ARTICLE

https://doi.org/10.1038/s41467-019-08464-y

\title{
The compound TB47 is highly bactericidal against Mycobacterium ulcerans in a Buruli ulcer mouse model
}

Yang Liu (1) 1,2, Yamin Gao 1,3, Jianxiong Liu4, Yaoju Tan4, Zhiyong Liu', Chiranjibi Chhotaray 1,3, Huofeng Jiang ${ }^{1,5}$, Zhili Lu1', Gift Chiwala1,3, Shuai Wang ${ }^{1,3}$, Gaelle Makafe (10 1,3, Md Mahmudul Islam¹,3, H.M. Adnan Hameed 1,3, Xingshan $\mathrm{Cai}^{4}$, Changwei Wang ${ }^{1}$, Xinjie $\mathrm{Li}^{4}$, Shouyong $\operatorname{Tan}^{4}$ \& Tianyu Zhang ${ }^{1,3}$

Buruli ulcer (BU) is an emerging infectious disease that causes disfiguring skin ulcers. The causative agent, Mycobacterium ulcerans, secretes toxin called mycolactone that triggers inflammation and immunopathology. Existing treatments are lengthy and consist of drugs developed for tuberculosis. Here, we report that a pyrazolo[1,5-a]pyridine-3-carboxamide, TB47, is highly bactericidal against $M$. ulcerans both in vitro and in vivo. In the validated mouse model of BU, TB47 alone reduces $M$. ulcerans burden in mouse footpads by more than $2.5 \log _{10}$ CFU compared to the standard BU treatment regimen recommended by the WHO. We show that mutations of ubiquinol-cytochrome $C$ reductase cytochrome subunit $B$ confer resistance to TB47 and the dissimilarity of CydABs from different mycobacteria may account for their differences in susceptibility to TB47. TB47 is highly potent against M. ulcerans and possesses desirable pharmacological attributes and low toxicity that warrant further assessment of this agent for treatment of BU.

\footnotetext{
${ }^{1}$ State Key Laboratory of Respiratory Disease, Guangzhou Regenerative Medicine and Health Guangdong Laboratory (GRMH-GDL), Guangzhou Institutes of Biomedicine and Health (GIBH), Chinese Academy of Sciences (CAS), Guangzhou 510530, China. ${ }^{2}$ Institute of Physical Science and Information Technology, Anhui University, Hefei 230601, China. ${ }^{3}$ University of Chinese Academy of Sciences (UCAS), Beijing 100049, China. ${ }^{4}$ State Key Laboratory of Respiratory Disease, Department of Clinical Laboratory, Guangzhou Chest Hospital, Guangzhou 510095, China. ${ }^{5}$ School of Life Sciences, University of Science and Technology of China, Hefei 230027, China. These authors contributed equally: Yang Liu, Yamin Gao. Correspondence and requests for materials should be addressed to T.Z. (email: zhang_tianyu@gibh.ac.cn)
} 
B uruli ulcer (BU) ${ }^{1-3}$, caused by Mycobacterium ulcerans, is the third prevalent mycobacterial disease, after tuberculosis (TB) and leprosy. The World Health Organization (WHO) recommends a combination of rifampin and streptomycin for 2 months for treatment of $\mathrm{BU}$ following evaluation of this regimen in a murine model of $\mathrm{BU}^{3}$. Although this treatment is suboptimal as streptomycin needs to be injected daily for at least 2 months and may result in hearing loss ${ }^{4,5}$, surgery that was previously the only possible therapeutic intervention became only adjuvant treatment for serious case. All potential oral regimens ${ }^{4,5}$ repurposed from TB treatment arsenal are not obviously more potent against $M$. ulcerans in vivo.

It takes $\sim 3$ months for $M$. ulcerans to form a visible colony on agar supplemented with enriched media specific for mycobacterial growth. Traditional methods that depend on enumerating colony-forming units of bacteria to evaluate efficacy of a drug would significantly prolong the duration of preclinical studies required for development of anti-BU treatment ${ }^{6,7}$. To address this concern, an autoluminescent reporter M. ulcerans strain $(\mathrm{AlMu})$, which spontaneously emits light without the addition of exogenous substrate was created using the luxCDABE operon from Photorhabdus luminescens and used for anti-BU drug discovery including for in vivo efficacy evaluation ${ }^{6}$. That study assessed activities of a panel of drugs with drugs with different mechanisms of actions and drug combinations and demonstrated that relative light units (RLU) counts correlated well with colony-forming unit (CFU) of M. ulcerans ${ }^{6}$. Use of this technology enabled rapid (requiring only $3 \mathrm{~s}$ ) and serial real-time monitoring of $M$. ulcerans in samples in vitro and in the same batch of small live animals for evaluation of drug activity. Therefore, this approach can drastically reduce the time, effort, animals, and resources necessary for in vitro and in vivo assessment that require monitoring of $M$. ulcerans growth. Here, we used AlMu to evaluate our small compounds library to discover agents to develop new anti-BU treatment.

We recently reported pyrazolo[1,5-a]pyridine-3-carboxamides exhibit activity against Mycobacterium tuberculosis ${ }^{8}$. The lead compound of this class, TB47, is now being developed as an anti-TB drug (http://www.newtbdrugs.org/pipeline/discovery). Its mechanism of action is unknown and agents with this pharmacophore have not been evaluated against non-tuberculous mycobacteria. In this study, we found TB47 to be highly potent against $M$. ulcerans $^{9}$. We also identified the target inhibited and the mechanism by which resistance to TB47 may arise.

\section{Results}

Bactericidal activity against M. ulcerans in vitro. We determined the minimum inhibitory concentrations (MICs) of TB47 against multiple non-tuberculous mycobacteria and a range of clinically important bacteria using standard broth dilution assay as per the Clinical and Laboratory Standard Institute Guidelines (Supplementary Table 1$)$. While its MICs $\left(\mu \mathrm{g} \mathrm{mL}^{-1}\right)$ were $>128$ for most of the clinical strains, the lowest MICs (0.0016) were observed for the two different $M$. ulcerans isolates. These data indicated that TB47 is specific to and highly potent against $M$. ulcerans. Next, we used the standard time kill assay to determine whether TB47 was bacteriostatic or bactericidal against M. ulcerans. In this assay, TB47 demonstrated bactericidal activity at concentration $\geq 0.0016 \mu \mathrm{g} \mathrm{mL}^{-1}$ (Fig. 1a). The bactericidal activity of TB47 at $0.008 \mu \mathrm{g} \mathrm{mL}^{-1}$ or higher was more potent than that exhibited by $0.2 \mu \mathrm{g} \mathrm{mL}{ }^{-1}$ rifampin, a drug currently used to treat BU. The bactericidal activity of TB47 was also verified by enumerating CFU (Supplementary Fig. 1). Its minimum bactericidal concentration (MBC) against M. ulcerans is $0.0064 \mu \mathrm{g} \mathrm{mL}^{-1}$.
In vivo bactericidal activity of TB47 against $M$. ulcerans. We evaluated activity of TB47 in a validated mouse model of BU $4,6,7$ using AlMu generated from M. ulcerans 10596. TB47 exhibited bactericidal activity even at $\geq 0.4 \mathrm{mg} \mathrm{kg}^{-1}$, the lowest among doses we evaluated in the mouse model of BU. TB47 alone at 0.8 $\mathrm{mg} \mathrm{kg}^{-1}$ exhibited efficacy superior to the current WHO recommended standard regimen to treat Buruli ulcer which includes a combination of 10 rifampin and 150 streptomycin (doses $\mathrm{mg} \mathrm{kg}^{-1}$ ) in the live mouse model (Fig. $1 \mathrm{~b}, P<0.001$ ). At doses $\geq 12.5 \mathrm{mg} \mathrm{kg}^{-1}$, the bioluminescence detected 3 days after treatment completion (at day 7) in live mice was indifferent from the background reading (Fig. 1b, $P>0.05$ ). The classical disease presentation in the mouse model of BU includes swelling, redness and tenderness of the footpads (the site of M. ulcerans injection). In mice treated with $\geq 3.1 \mathrm{mg} \mathrm{kg}^{-1} \mathrm{~TB} 47$, at day 7 , we observed no swelling or redness in the footpads of mice with average lesion index ${ }^{10}$ (ALI) $=0$, while those treated with the standard regimen containing rifampin and streptomycin exhibited $\mathrm{ALI}=3$ (Fig. 2). Alternatively, we used bioluminescence and CFUs to quantify M. ulcerans burden in the footpad tissue at day 7. This assessment also showed $>2 \log _{10}$ reduction in RLU in the footpad tissue of mice treated with $\geq 3.1 \mathrm{mg} \mathrm{kg}^{-1} \mathrm{~TB} 47$. The minimal bactericidal dose (MBD) was $1.6 \mathrm{mg} \mathrm{kg}^{-1}$ and at doses $\geq 12.5 \mathrm{mg} \mathrm{kg}^{-1}$, RLUs were reduced to the background level. M. ulcerans burden (CFU per footpad) in mice treated with $50 \mathrm{mg} \mathrm{kg}^{-1}$ of TB47 was $3.9 \log _{10}$ lower than that at day 0 and $\sim 2.5 \log _{10}$ lower than in mice treated with the standard regimen containing rifampin and streptomycin (Fig. 1c).

Preliminary sterilizing activity. For mice treated with $\geq$ $25 \mathrm{mg} \mathrm{kg}^{-1}$ of TB47, RLU could not be detected at significant levels even up to 14 weeks post completion of treatment (Fig. 1d). However, M. ulcerans infection relapsed in 4 to 6 out of 10 mice at 20 to 24 weeks (ALI > 1, Supplementary Fig. 2). In a separate experiment, mice treated with $25 \mathrm{mg} \mathrm{kg}^{-1} \mathrm{~TB} 47$ for 1 or 2 weeks were compared with mice treated with the standard regimen containing rifampin and streptomycin for 5 or 6 weeks. RLUs from footpads of live mice treated with the standard regimen for 4 weeks were still above the background reading, while the RLUs from footpads of live mice treated with TB47 for 5 days reached the background noise levels (Fig. 1e). In comparison to the standard regimen for 5 weeks, $25 \mathrm{mg} \mathrm{kg}^{-1} \mathrm{~TB} 47$ for 1 week significantly extended the time-to-replase $(P<0.01$; Fig. 1f). Also, longer duration of treatment of TB47 further prolonged time-to-relapse.

Resistance to TB47 due to QcrB. To explore its mechanism of action, Mycobacterium marinum, a close relative of $M$. ulcerans with $>98 \%$ genome sequence identity ${ }^{2}$, was used at first as it takes only about 2 weeks for $M$. marinum to form a visible colony on the agar plate. We were able to select $M$. marinum spontaneous mutants in the presence of TB47 and confirmed that the isolates stably exhibited resistance to TB47 (Fig. 3a). The isolates exhibited a consistent increase in MICs for TB47 but remained susceptible to the control drug rifampin (Table 1). Similarly, nine TB47resistant $M$. ulcerans colonies were isolated very recently only on plates containing $0.02 \mu \mathrm{g} \mathrm{mL}^{-1} \mathrm{~TB} 47$ but none on plates containing $\geq 0.05 \mu \mathrm{g} \mathrm{mL}^{-1} \mathrm{~TB} 47$, although we attempted $>10$ screens at $\geq 0.05 \mu \mathrm{g} \mathrm{mL}^{-1}$ of TB47. Based on three independent mutantselection attempts using $M$. marinum and $M$. ulcerans, we determined spontaneous resistance mutation rates against TB47 to be $0.45 \times 10^{-8}$ for $M$. marinum at $1 \mu \mathrm{g} \mathrm{mL}^{-1}$ and $0.83 \times 10^{-9}$ for M. ulcerans at $0.02 \mu \mathrm{g} \mathrm{mL}-1$.

We sequenced the genomes of seven independent TB47resistant $M$. marinum isolates and the parent strain. We identified single-nucleotide polymorphisms (SNPs) at the same codon in 
a

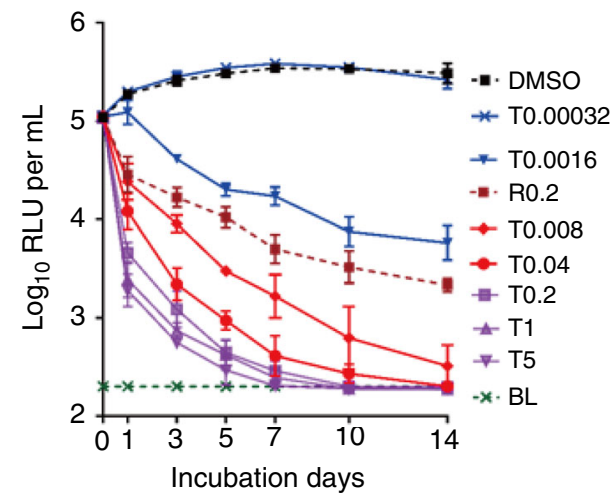

b

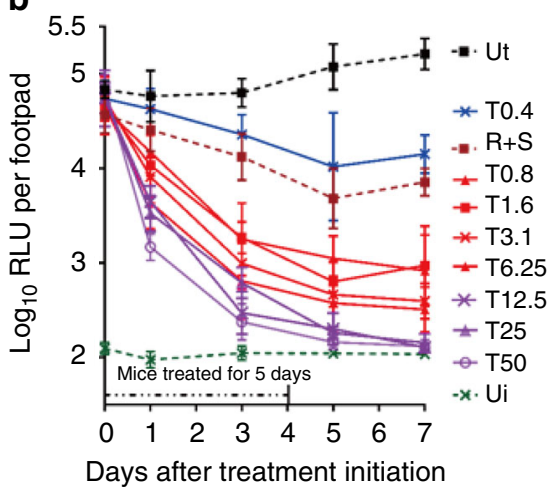

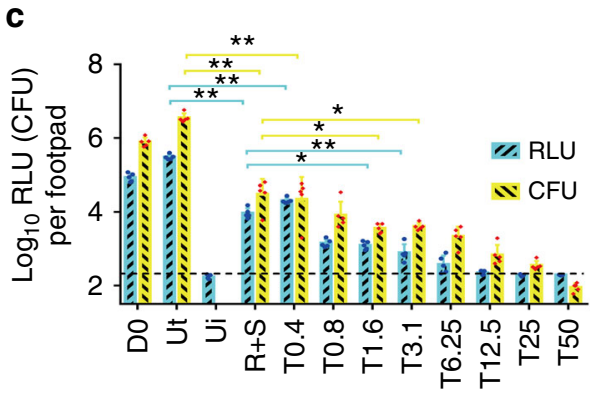

Different treatments

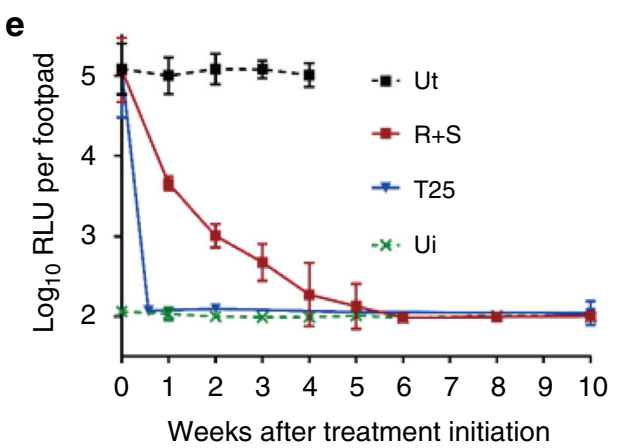

d
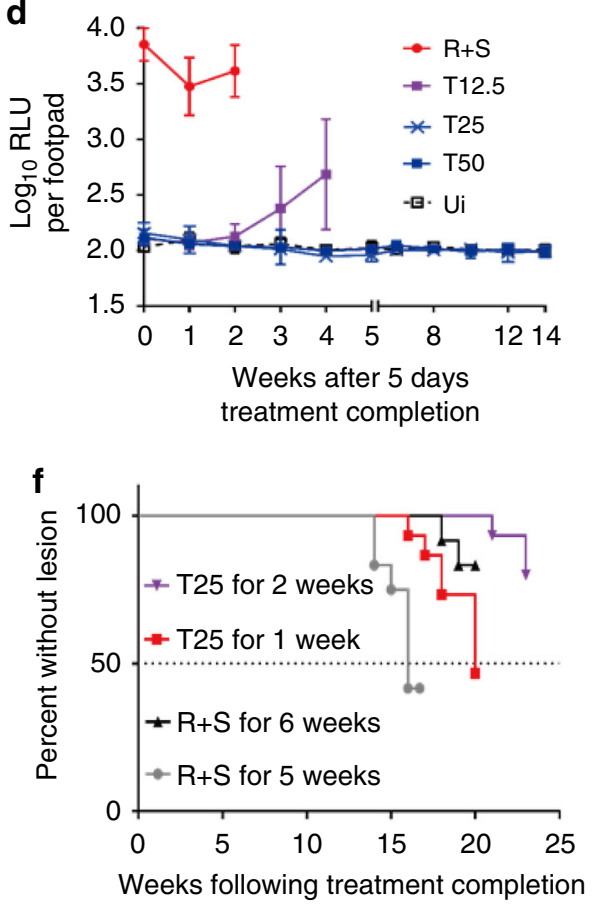

Fig. 1 Activity of TB47 (T) against M. ulcerans. a Time-killing curves of TB47 against AIMu in liquid culture. Concentrations ( $\mu g \mathrm{~mL}^{-1}$ ). Data are expressed as mean \pm SD from three independent biological repeats. The experiment was performed in triplicate (three independent experiments) and the representative results are shown. b-d Mice were treated from 12 days post infection of AIMu (ALI =1.09 \pm 0.28$)$. Data are expressed as mean \pm SD of five samples. b RLUs detected from left hind footpads of the same batch of live mice treated for 5 days and stopped for 3 days. Statistical analysis was performed using unpaired Student's $t$-test. c RLUs and CFUs of footpad tissue suspension at day 0 and day 7 . The experiments for $\mathbf{b}, \mathbf{c}$ were performed in triplicate (three independent experiments) and the representative results are shown. The correlation coefficients $\left(R^{2}\right)$ of RLU and CFU for the in vivo experiments were 0.966 (including the positive) and 0.898 (TB47 treated groups only), respectively. Correlation analysis was performed using Pearson's correlation test. The linearity of the relationship of RLU and CFU for the footpad suspension is valid when the CFUs are from 2.5 to 6.2 log 10 CFU mL ${ }^{-1}$. Statistical analysis was performed using unpaired Student's $t$-test, ${ }^{\star} P<0.001 ;{ }^{\star \star} P<0.0001$. d Footpad RLUs detected after completion of treatment for monitoring relapse. e Kinetic curves of RLUs from the footpads of live mice treated with different regimens. Data are expressed as mean \pm SD of five samples. $\mathbf{f}$ Time to footpad swelling after completion of antibiotic treatment. Time to footpad swelling in mice treated with either rifampin + streptomycin for 5 weeks (gray circles) or 6 weeks (black triangles), or TB47 for 1 week (red square) or for 2 weeks (purple triangle). Statistical differences were determined by Log-rank (Mantel-Cox) test with 15 mice in each group. T, TB47; BL, base line; Ui, uninfected (for RLU detection, Ui is the base line); Ut, untreated; R, rifampin, 25; S, streptomycin, 150; Dosage $\left(\mathrm{mg} \mathrm{kg}^{-1}\right)$. The dotted green lines indicated the base line (the limit of detection). Data are expressed as mean \pm SD. ${ }^{*} P<0.001 ;{ }^{*} P<0.0001$

all resistant isolates $\mathrm{ACC} \rightarrow \mathrm{GCC}$ or CCC resulting in Thr323Ala or Thr323Pro substitution in QcrB (ubiquinol-cytochrome C reductase cytochrome subunit $\mathrm{B}$ ) (Table 1). We amplified this locus in an additional 20 independent spontaneous M. marinum mutants, sequenced and found mutations only in the same codon that resulted in Thr323Ala or Thr323Pro substitutions (Table 1 and Fig. 3b). Alignment of amino acid sequences of QcrBs from $M$. ulcerans and M. marinum revealed $100 \%$ identity
(Supplementary Fig. 3a). Similarly, we sequenced $q c r B$ gene in 9 independent spontaneous $M$. ulcerans mutants. Eight out of nine mutants harbored the SNP ACC $\rightarrow$ GCC resulting in Thr323Ala while the remaining isolate harbored SNP ACC $\rightarrow$ ATC resulting in Thr323Ile. To verify if such substitutions truly lead to resistance to TB47, we overexpressed wild-type and mutated $q c r B$ genes in $M$. marinum and $M$. ulcerans, and observed an increase in MICs of TB47 (Table 1). Based on these data, we 


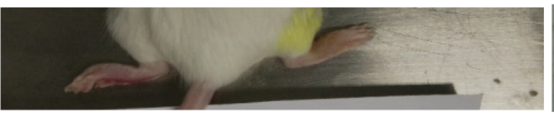

Before treatment

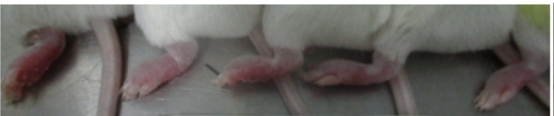

Untreated

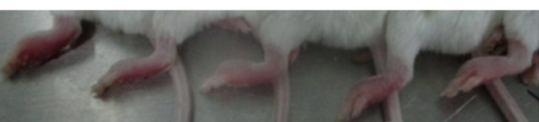

$R 10+S 150$

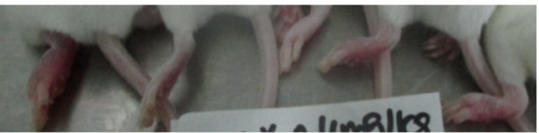

T 0.4

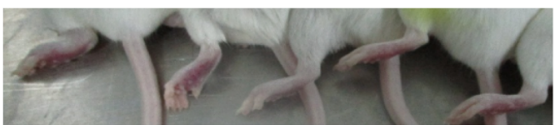

T 0.8

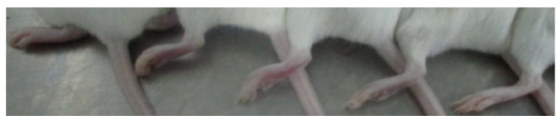

$\mathrm{T} 1.6$

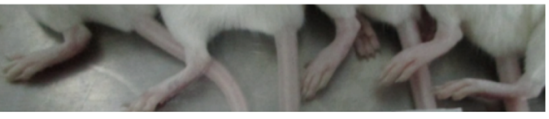

T 3.1

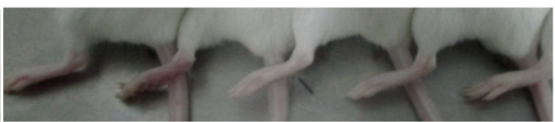

T 6.25

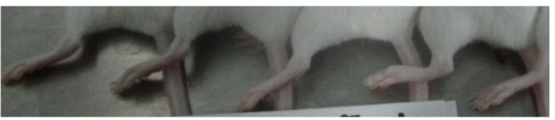

$\mathrm{T} 12.5$

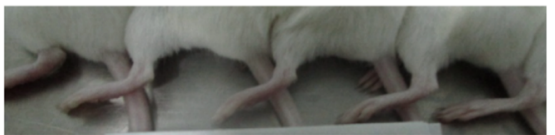

T 25

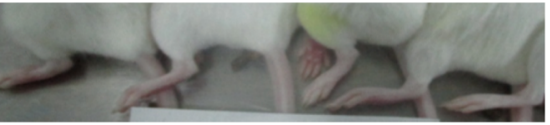

T 50

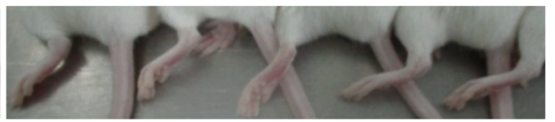

Uninfected

Fig. 2 Photographs showing the swelling degrees of the left hind mouse footpads at 0 and day 7 . The $A L I$ is $3 \pm 0,2.8 \pm 0.27$, and $1.8 \pm 1.25$, for the untreated, rifampin + streptomycin and T0.4, respectively. Only one obvious swelling footpad (swelling index about 2) was observed in the T0.8 mg kg ${ }^{-1}$ group (the left one) and one questionable swelling footpad in the $T 0.8 \mathrm{mg} \mathrm{kg}^{-1}$ group (the middle one) and no any swelling footpad (ALI = 0 ) was observed in any groups treated with $T \geq 3.1 \mathrm{mg} \mathrm{kg}^{-1}$. T, TB47; R, rifampin; $\mathrm{S}$, streptomycin. Dosage, $\mathrm{mg} \mathrm{kg}^{-1}$

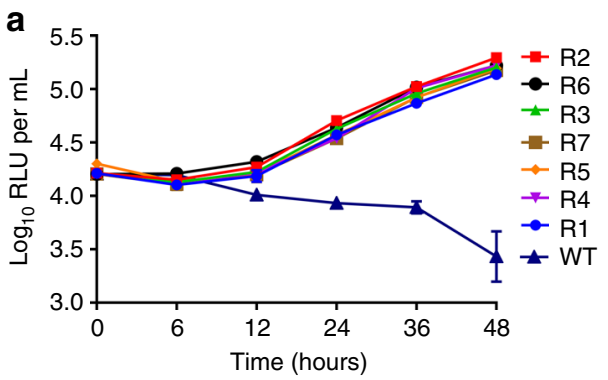

b

- R2 312 GSQPDFYMMWPEGLARIWPP 332

- R6 312 GSQPDFFMMWAEGLARIWPP 332

- R3 312 GSOPDFYMMWPEGLARIWPP 332

- R7 312 GSQPDFYMMWAEGLARIWPP 332

- R5 312 GSQPDFYMMWPEGLARIWPP 332

- R4 312 GSQPDFYMMWAEGLARIWPP 332

- R1 312 GSQPDFYMMWPEGLARIWPP 332

- WT 312 GSOPDFYMMWTEGLARIWPP 332

Fig. 3 Drug susceptibility testing and sequence analysis of resistant mutants. a Time-killing cures of TB47 ( $\left.1 \mu \mathrm{g} \mathrm{mL}^{-1}\right)$ against autoluminescent $M$. marinum resistant mutants (R1 to R7) and the parent strain in liquid culture. Data are expressed as mean \pm SD from three independent biological repeats. The experiment was performed in triplicate (three independent experiments) and the representative results are shown. b Results of polymorphism in QcrB conferring resistance to TB47. WT, wild-type

conclude that mutations of QcrB Thr323 confer M. marinum and M. ulcerans resistance to TB47.

Two terminal oxidases in electron transport chain. The electron transport chain of mycobacteria usually contains two terminal oxidases, the cytochrome bcl:aa3 containing QcrCAB and the cytochrome bd oxidase (Cyt-bds) containing CydAB (Fig. 4). Inhibition of the bcl:aa3 complex is only bacteriostatic because the alternate Cyt-bds is capable of maintaining a membrane potential and menaquinol oxidation and is therefore sufficient to maintain respiration to protect $M$. tuberculosis from death ${ }^{11}$. So if TB47 inhibits QcrB, it should be more powerful against the strain lacking of functional Cyt-bds. The $c y d A B$ genes were deleted in the model organism $M$. smegmatis to generate a $\triangle c y d A B$ strain $($ Msm $\triangle c y d A B)$. The synthetic lethal interaction between the Cyt-bc1:aa3 and the Cyt-bds was evaluated by treating the Msm $\triangle c y d A B$ with TB47. A synthetic lethal interaction is a well-described phenomenon where simultaneous inactivation of two genes that confer an essential activity results in cell death but inactivation of either gene alone does not affect cell viability. Similar to a synthetic lethality described in M. tuberculosis $^{11}$, deletion of $c y d A B$ in $M$. smegmatis did not impact significantly on bacterial growth (Supplementary Fig. 4) but sharply increased the inhibitory potency of TB47 (Fig. 5a) as its MIC was reduced from 50 to $3.12 \mu \mathrm{g} \mathrm{mL}^{-1}$.

Activities of Cyt-bds from different mycobacteria. The amino acid sequences of QcrB, CydA, and CydB from different mycobacteria were aligned (Supplementary Fig. 3a, b, c). The QcrB as shown before ${ }^{12}$, is highly conserved among mycobacteria, but 
Table 1 Susceptibilities of different M. marinum/M. ulcerans strains to TB47 and rifampin in liquid using bioluminescence

\begin{tabular}{|c|c|c|c|}
\hline $\begin{array}{l}\text { Mycobacteria } \\
\text { M. marinum strains }\end{array}$ & Gene: qcrB from $M$. marinum & \multicolumn{2}{|c|}{$M I C_{\text {lux }} s\left(\mu \mathrm{g} \mathrm{mL}^{-1}\right)$} \\
\hline Wild-type (Autoluminescent strain) & Wild-type & $0.001-0.008$ & $0.25-1.0$ \\
\hline Type 2 mutant & ACC to GCC (Thr323Ala) & $>128$ & $0.25-1.0$ \\
\hline Wild-type:: vector & Wild-type containing an empty plasmid & $0.001-0.008$ & $0.25-1.0$ \\
\hline Wild-type:: qcrBWT & Over-expression of wild-type $q c r B$ & $32-64$ & $0.25-1.0$ \\
\hline M. ulcerans strains & & TB47 & RIF \\
\hline Wild-type (AIMu 1059) & Wild-type & 0.0016 & $0.06-0.25$ \\
\hline Wild-type:: vector & Wild-type containing an empty plasmid & $0.0016-0.0031$ & $0.06-0.25$ \\
\hline Wild-type:: qcrBWT & Over-expression of wild-type $q c r B$ & $0.05-0.10$ & $0.06-0.25$ \\
\hline Wild-type:: qcrB ${ }^{\text {THR323PRO }}$ & Over-expression of $q c r B^{T h r 323 P r o}$ & 0.10 to 0.40 & $0.06-0.25$ \\
\hline Wild-type:: qcrB ${ }^{\text {THR323ALA }}$ & Over-expression of $q c r B^{T h r 323 A l a}$ & 0.10 to 0.40 & $0.125-0.5$ \\
\hline
\end{tabular}

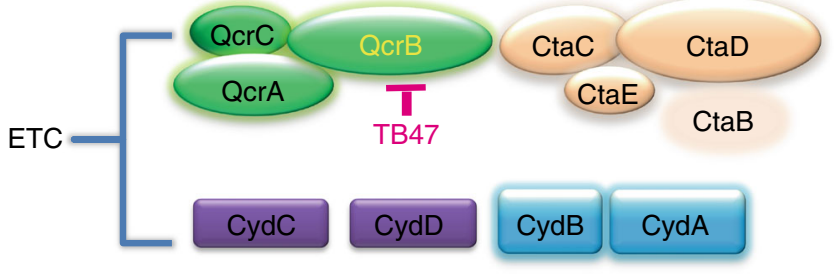

Fig. 4 Two terminal oxidases in the electron transport chain of mycobacteria. Mutations in QcrB in the cytochrome bc1:aa3 confer resistance to TB47

the similarity of CydA/B between $M$. tuberculosis and that of M. smegmatis or M. ulcerans was low. It is interesting that M. leprae genome altogether lacks genes that encode CydA/B. This led us to hypothesize that the Cyt-bds from different mycobacteria may not have identical activities, which potentially explained the distinct susceptibilities of mycobacteria to TB47.

To verify this hypothesis, we created a series of recombinant strains by introducing $c y d A B$ genes from different mycobacteria into Msm $\triangle c y d A B$. The MICs of TB47 against Msm $\triangle c y d A B:$ : $c y d A B^{M s m}, \operatorname{Msm} \triangle c y d A B:: c y d A B^{M t b}, \operatorname{Msm} \triangle c y d A B:: c y d A B^{M u}$, and Msm $\triangle c y d A B$ were $50,37.5,6.5$, and $3.2 \mu \mathrm{g} \mathrm{mL}^{-1}$, respectively (Fig. 5a), while MICs of isoniazid were $4 \mu \mathrm{g} \mathrm{mL}^{-1}$ for all four strains. In the presence of TB47, these strains exhibited different in vitro growth profiles (Fig. 5b). M. ulcerans cydAB could not sufficiently complement the function of $M$. smegmatis Cyt-bds. This indicated that intrinsic activity of Cyt-bds of M. ulcerans could be too weak, which may explain well why TB47 had surprising bactericidal activity against $M$. ulcerans.

Pharmacological attributes and low toxicity of TB47. There is no obvious toxicity of TB47 observed until now. TB47 did not display obvious cytotoxicity with $\mathrm{IC}_{50}$ of $>100 \mu \mathrm{M}$ for VERO cell and $50 \mu \mathrm{M}$ for THP-1 cell. TB47 did not inhibit hERG $\left(\mathrm{IC}_{50}>30 \mu \mathrm{M}\right)$, suggesting a low risk for cardiotoxicity. A single oral administration of $2000 \mathrm{mg} \mathrm{kg}^{-1}$ of TB47 or oral $200 \mathrm{mg} \mathrm{kg}^{-1}$ daily for 4 weeks, the highest dosage we tested, was well tolerated by mice. No genotoxicity or obvious CYP inhibition was associated with this compound, and its metabolic stability in different animal species and permeability are high (Supplementary

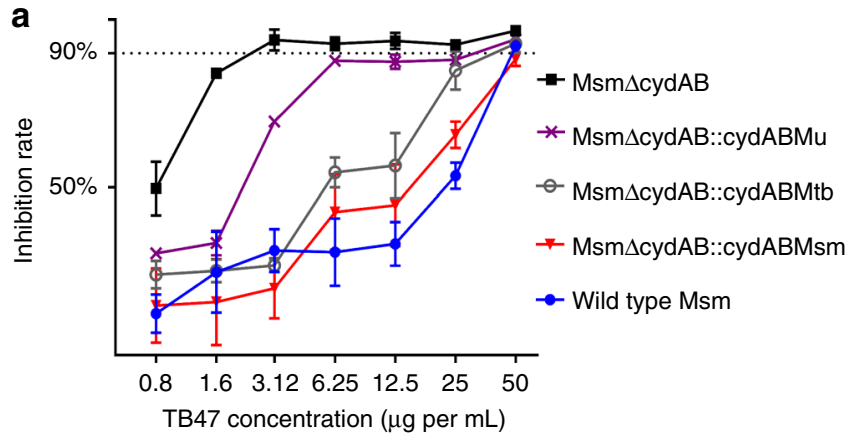

b

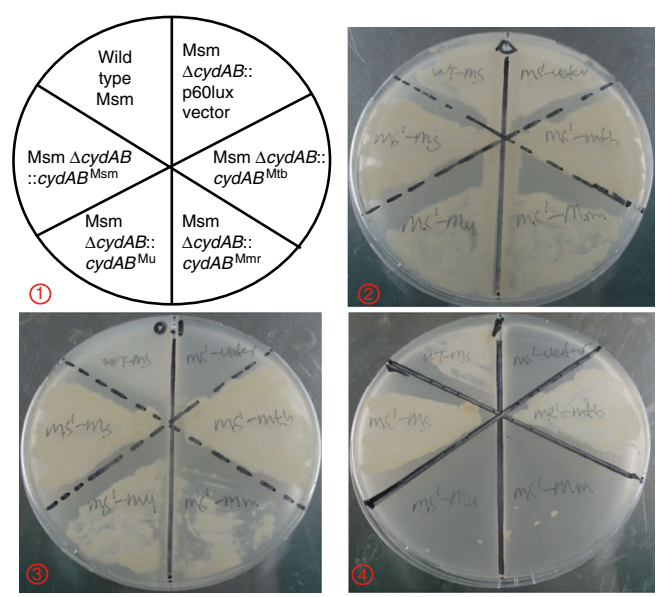

Fig. 5 Effect on the sensitivity to TB47 during the over-expression of CydABs in M. smegmatis. a The inhibition rates against $M$. smegmatis $\mathrm{mc}^{2}$ 155 and derivative strains. Bacteria treated with different concentrations of TB47 and fluorescence units (FU) were recorded $4 \mathrm{~h}$ post-alamar blue addition. The inhibition rate was calculated as $\mathrm{FU}_{\text {treatment }} / \mathrm{FU}_{\text {untreated }} \times$ $100 \%$ and recorded for each strain per treatment. Data are expressed as mean \pm SD from three independent repeats. $\mathbf{b}$ Inhibition growth of different M. smegmatis strains by TB47 at different concentrations. (1) The plate layout is shown (M. smegmatis containing the construct detailed). (2) to (4) showing the plates containing TB47 at $0,0.1$, and $1\left(\mu \mathrm{gL}^{-1}\right)$ incubated at $37^{\circ} \mathrm{C}$ for 36 h. Msm, M. smegmatis; Mu, M. ulcerans; Mtb, M. tuberculosis; Mmr, M. marinum 


\begin{tabular}{|c|c|c|c|c|}
\hline Drug delivering & Parameters & Unit & Mean & SD \\
\hline \multirow[t]{8}{*}{ Intravenous injection } & $A \cup C_{(0-t)}$ & $\mu g L^{-1} \times h$ & 10,409 & 139 \\
\hline & $\mathrm{AUC}_{(0-\infty)}$ & $\mu g L^{-1} \times h$ & 11,951 & 565 \\
\hline & $\mathrm{MRT}_{(0-t)}$ & $\mathrm{h}$ & 14.7 & 1.7 \\
\hline & $\mathrm{MRT}_{(0-\infty)}$ & $\mathrm{h}$ & 22.3 & 3.7 \\
\hline & $t_{1 / 2 z}$ & $\mathrm{~h}$ & 17.7 & 2.6 \\
\hline & $\mathrm{CL}$ & $\mathrm{L} \mathrm{h}^{-1} \mathrm{~kg}^{-1}$ & 0.17 & 0.01 \\
\hline & V & $\mathrm{L} \mathrm{kg}^{-1}$ & 4.27 & 0.44 \\
\hline & $C_{2 \min }$ & $\mu g L^{-1}$ & 3709 & 1805 \\
\hline \multirow[t]{9}{*}{ Oral administration } & $A \cup C_{(0-t)}$ & $\mu \mathrm{g} \mathrm{L}^{-1} \times \mathrm{h}$ & 19,823 & 1665 \\
\hline & $\mathrm{AUC}_{(0-\infty)}$ & $\mu g L^{-1} \times h$ & 33,144 & 5164 \\
\hline & $\mathrm{MRT}_{(0-t)}$ & $\mathrm{h}$ & 20.2 & 0.3 \\
\hline & $\mathrm{MRT}_{(0-\infty)}$ & h & 52.0 & 4.2 \\
\hline & $t_{1 / 2 z}$ & $\mathrm{~h}$ & 35.6 & 2.7 \\
\hline & $T_{\max }$ & $\mathrm{h}$ & 3.2 & 2.8 \\
\hline & $C L$ & $\mathrm{~L} \mathrm{~h}^{-1} \mathrm{~kg}^{-1}$ & 0.30 & 0.02 \\
\hline & V & $\mathrm{L} \mathrm{kg}^{-1}$ & 15.49 & 0.38 \\
\hline & $C_{\max }$ & $\mu g \mathrm{~L}^{-1}$ & 626 & 282 \\
\hline
\end{tabular}

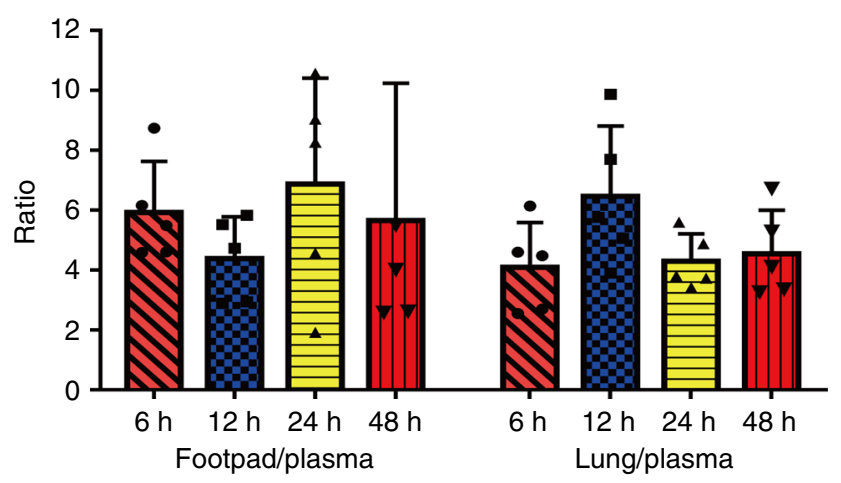

Fig. 6 The ratios of footpad/plasma and lung/plasma of TB47 concentrations. BALB/c mice were given a dose of $10 \mathrm{mg} \mathrm{kg}^{-1} \mathrm{~TB} 47$ by oral gavage. Data are expressed as mean \pm SD of five samples

Table 2). Furthermore, in a long-term administration study, TB47 was well tolerated without any meaningful clinical signs of toxicity when administered daily at a dose of 10,30 , or $100 \mathrm{mg} \mathrm{kg}^{-1}$ body weight orally for 30 days using both male and female rats. These data demonstrate that TB47 is well tolerated even during prolonged use.

Pharmacokinetic parameters of TB47 in BALB/c mice are summarized in Table 2. After intravenous administration of $2 \mathrm{mg} \mathrm{kg}^{-1} \mathrm{~TB} 47$, the AUC $(0-t)$ and $t_{1 / 2}$ were $10,409 \pm$ $139 \mu \mathrm{g} \mathrm{L}^{-1} \times h$ and $17.7 \pm 2.6 \mathrm{~h}$, respectively. Whereas the AUC $(0-t), C_{\max }$ and $t_{1 / 2}$ were $19,823 \pm 1665 \mu \mathrm{g} \mathrm{L}{ }^{-1} \times h, 0.63 \pm$ $0.28 \mu \mathrm{g} \mathrm{mL}^{-1}$ and $35.6 \pm 2.7 \mathrm{~h}$, respectively, after oral administration of $10 \mathrm{mg} \mathrm{kg}^{-1} \mathrm{~TB} 47$, and the bioavailability was $38.1 \%$. The long half-life and high blood concentration of TB47 are likely significant contributors to its potent in vivo activity against $M$. ulcerans. Furthermore, the concentrations in mice foot tissue or lungs at $6,12,24$, and $48 \mathrm{~h}$ were $>4$ times higher than in the plasma (Fig. 6).

\section{Discussion}

Despite the the efficacy of the standard regimen of streptomycin and rifampin for 2 months for treatment of BU, it has significant disadvantages including daily parenteral administration and potential risk of side effects, such as hearing loss ${ }^{4,5}$. An all-oral and less toxic treatment regimen of Buruli ulcer has been sought after and encouraged by WHO. However, most of newly developed treatments were based on rifampin although it is well known that rifampin causes interactions with many drugs, including anti-retroviral agents and rifampin-resistance in $M$. ulcerans isolates from patients and infected animals have been reported $^{7,13}$. New powerful drugs, especially those with new mechanisms of action could address the current need for novel regimens to treat $\mathrm{BU}$.

TB47 exhibits potent bactericidal activity with very low MIC and MBC against $M$. ulcerans. Detection of MICs of TB47 against M. ulcerans on agar plates was repeated only once (duplicate but not triplicate), which may hinder potential reproducibility. However, We (1) used two strains (M. ulcerans 1059 and M. ulcerans 1615) and they showed the same MICs of TB47 and the MIC detection was duplicated for each strain; (2) repeated the liquid $\mathrm{MIC}_{\mathrm{lux}}$ detection more than four times by different persons; (3) repeated more than ten times to select TB47resistant $M$. ulcerans mutants on agar containing as low as 0.05 to $0.1 \mu \mathrm{g} \mathrm{mL}-1$ TB47 but failed. This observation further validates the very low MIC observed for TB47;(4) repeated the MBC determination three times (independent repeats) and confirmed it too is very low. Moreover, it is striking that even at $0.8 \mathrm{mg} \mathrm{kg}^{-1}$ of TB47, once daily, it exhibited bactericidal activity and overall superior efficacy than the currently used standard regimen containing rifampin and streptomycin. While mice treated with the standard regimen sustained clinical indications such as swelling of the footpad, such indications were nonexistent in the footpads of mice that received TB47 at doses of $\geq 3.1 \mathrm{mg} \mathrm{kg}^{-1}$. In addition, in comparison to the standard regimen, TB47 prolonged the time-to-relapse. This indicated that TB47 has a quick therapeutic effect and could possibly shorten the duration of the BU treatment. Although TB47 alone showed very good activity against M. ulcerans, it did not produce $100 \%$ cure at $25 \mathrm{mg} \mathrm{kg}^{-1}$ daily, 5 days per week, for 2 weeks.

We demonstrate that TB47 targets mycobacterial QcrB because QcrB mutations cause TB47 resistance and deletion of cytochrome bd oxidase (Cyt-bds) makes mycobacteria more susceptible to TB47 (Fig. 4). The differences in Cyt-bds from different bacteria potentially explain their differential susceptibilities to TB47. The M. smegmatis $\triangle c y d A B$ can be a good system to test the function of $c y d A B$ introduced from other mycobacteria and could be used as a model for screening inhibitors of Cyt-bds. It is valuable to test activity of Cyt-bds in different mycobacteria, such as $M$. leprae. There are no significant similar $\mathrm{CydAB}$ proteins in M. leprae, which indicated that Cyt-bcl:aa3 could be the only terminal oxidase in the electron transport chain in this mycobacterium. So it is very attractive to test TB47 against leprosy caused by $M$. leprae as it is still a public health concern in many countries despite sustained efforts to eliminate it over the last 30 years. Depending upon the leprosy patient's disease presentation, they are currently treated for 6,12 , or 24 months with a combination of rifampin, dapsone and with or without clofazimine ${ }^{14}$. Many patients experience side effects, toxicity, and potential drug resistance ${ }^{15,16}$. Our study also supports that the combination of the potential Cyt-bds inhibitors and TB47 may shorten the duration of further for both drug-susceptible and drug-resistant TB. None of atomic structures of mycobacterial bd oxidases has been reported yet but that from Geobacillus thermodenitrificans was resolved recently ${ }^{17}$. The structures of mycobacterial $\mathrm{CydAB}$ complexes and the rational design of inhibitors of them are highly needed in the discovery and development of antimycobacterial drugs.

In summary, our study demonstrates that TB47 acts by a different mechanism compared to drugs considered to treat BU thereby reducing the chances of development of cross-resistance. In addition, the rate of spontaneous resistance of $M$. ulcerans to 
TB47 is very low. Oral bioavailability, relatively long half-life and lack of toxicity are additional appealing features of TB47. To leverage these clinically desirable attributes, we propose that a multi-drug combination regimen containing TB47 has promise to produce a stable cure while also potentially shortening the duration of treatment. Further preclinical assessments of dose, frequency and duration of administration would be necessary to harness the optimal potential of TB47 against BU.

\section{Methods}

Drugs formulation. TB47 was synthesized in a batch and supplied by Guangzhou Eggbio Co. Ltd with purity $98.67 \%$ by High Performance Liquid Chromatograph (Batch number: TB47160616). It was formulated in 0.05\% CMC-Na (sodium salt of carboxy methyl cellulose) for in vivo study. Rifampin and streptomycin were both bought from TCI (Japan) and dissolved in distilled water for in vivo use. Streptomycin and isoniazid (Macklin, Shanghai) were dissolved in distilled water, and rifampin and TB47 were dissolved in dimethyl sulfoxide (DMSO) for in vitro use. All drugs prepared weekly for in vivo studies. Hygromycin was bought from Roche (Swiss) in liquid form and kanamycin was bought from Sigma (USA). They were dissolved in distilled water for in vitro study.

Strains and growth conditions. M. ulcerans strains 1059 and 1615, M. marinum, M. smegmatis $\mathrm{C}^{2} 155$, and derivative strains were grown in Middlebrook $7 \mathrm{H} 9$ broth medium with $10 \% 2$-oxo-acid dehydrogenase complexes (OADC) $+0.2 \%$ glycerol $+0.05 \%$ tween 80 for culture and without tween 80 for drug susceptibility testing or on $7 \mathrm{H} 11$ plates supplemented with $0.2 \%$ glycerol $+10 \%$ OADC. M. ulcerans and M. marinum strains were grown at 30 to $32^{\circ} \mathrm{C}$ and others at $37^{\circ} \mathrm{C}$. Other clinical important bacteria were grown according to the Clinical and Laboratory Standard Institute Guidelines.

RLU-based MIC $C_{\text {lux }}$ and CFU-based MBC determination. Serial dilutions of drugcontaining solutions and autoluminescent mycobacteria broth culture $\left(\mathrm{OD}_{600}\right.$ of 0.3 to 1.0 ) were prepared ${ }^{6}$. RLU counts from the same batch of triplicate samples were measured according to the designed time points. Ninety-six-well plates were measured as rendering the amount of light by using a Veritas ${ }^{\mathrm{m}}$ Microplate Luminometer Operating Manual (Turner BioSystems). MIC lux was determined as the lowest concentration that can inhibit > 90\% RLUs compared with that from the untreated controls ${ }^{6,18}$. The time-killing curves and $\mathrm{MIC}_{\text {lux }}$ of autoluminescent strains were determined by detecting RLUs from these samples. For M. ulcerans and M. marinum strains, the incubation time for $\mathrm{MIC}_{\text {lux }}$ reading was 7 days and 3 days, respectively. The minimum bactericidal concentration (MBC) was defined as the lowest concentration of drug that killed $\geq 99 \%$ of the CFU compared with the CFU count of the initial inoculum used for the liquid activity assays, which was adopted from reference ${ }^{19}$. In our case, the liquid media in the culture tubes that showed the RLU at least $1 \log _{10}$ lower than that at day 0 were diluted and plated on 7H11 agar (supplemented with $10 \%$ OADC) plates for CFU counts. The concentrations used were twofold series of dilutions for this purpose. The incubation time for RLU reading was 14 days for the MBD experiment and the light from each $1.5 \mathrm{~mL}$ tube containing total $500 \mu \mathrm{L}$ culture was detected using Promega GloMax 20/20n. Triplicate tubes for each concentration were plated for CFU counts at $\mathrm{d} 0,3$, and 7 and to detect the quick bactericidal activity of TB47 and to show the correlation of RLU and CFU counts. The concentrations used were fivefold series of dilutions for this purpose. Each above experiment was performed in triplicate (three independent experiments) or more by independent persons and from three or more independent cultures. The representative results are shown.

MIC determination. The serial tenfold diluted mid-log-phase cultures were plated on $7 \mathrm{H} 11$ plates containing different concentrations of drugs. The MICs of TB47 to M. ulcerans strains 1059 and 1615 were defined as the lowest concentration that can inhibit at least $99 \%$ growth observed from drug-free control plates ${ }^{7}$ The experiments detecting such MICs using agar plates were only repeated once (in duplicate) and each time with two different strains. The results were shown as indicated in Supplementary Table 1. MICs against a range of clinically importan bacteria were tested using standard broth dilution assay as per the Clinical and Laboratory Standard Institute Guidelines.

Animal studies. All animal procedures were conducted according to relevant national and international guidelines. All animal care and experimental protocols were approved by the Committee on Laboratory Animal Ethics of Guangzhou Institutes of Biomedicine and Health (GIBH), Chinese Academy of Sciences, \#2017077. Serial, non-invasive, real-time monitoring of drug activity in a murine model of Buruli ulcer was used. Colony suspensions of autoluminescent M. ulcerans 1059 were made by vortex using $30 \mathrm{mg}$ of fresh colonies in $10 \mathrm{~mL}$ PBS and the resulting suspension was used to inject the left hind footpads of six-weekold, female BALB/c mice. The inoculum volume was $0.05 \mathrm{~mL}$, containing approximately 5 to $6 \log _{10} \mathrm{CFU}$. The right hind footpads served as negative controls for observation of swelling. The lesion index was defined as follows: index $0=$ normal footpad; index $1=$ noninflammatory footpad swelling; index $2=$ inflammatory footpad swelling; index 3 = inflammatory hind foot swelling ${ }^{10}$. The schemes for testing TB47 activity alone and in combination were demonstrated in Supplementary Table 3 and Supplementary Table 4, respectively. Subcutaneous route for streptomycin and oral gavage for others were used.

MBD detection. The MBD is defined as the lowest dose able to reduce the footpad CFU counts by $99 \%$ compared to the counts in the controls pretreatment, adopted from references ${ }^{20,21}$. Mice were treated for 5 days and sacrificed at $\mathrm{d} 7$ as demonstrated in Supplementary Table 3. This experiment was carried out more than triplicate by detecting footpad suspension and only footpad tissue from two independent experiments were plated for $\mathrm{CFU}$ counts at $\mathrm{d} 0$ and $\mathrm{d} 7 \mathrm{for} \mathrm{MBD}$ detection. The representative results are shown in Fig. 1c.

Selection of spontaneous-resistant mutants to TB47. Broth cultures $\left(\mathrm{OD}_{600}\right.$ from 0.6 to 1.3 ) of autoluminescent $M$. ulcerans 1059 , wild-type $M$. ulcerans 1615 , M. ulcerans 1059 , and autoluminescent M. marinum were plated on $7 \mathrm{H} 11$ plates containing $0.02,0.05,0.1,0.5,1,2,10$, or $40 \mu \mathrm{gL}^{-1} \mathrm{~TB} 47$. The colonies grown upon the TB47-containing plates were picked up to confirm the drug resistance phenotype by real-time in vitro drug susceptibility testing in liquid media or agar method as described above. We repeated about ten times using M. ulcerans and each time we plated at least 20 plates $(0.5 \mathrm{~mL}$ per plate, so $>10 \mathrm{~mL}$ culture per passage) at concentrations $\geq 0.1 \mu \mathrm{g} \mathrm{mL}^{-1}$ but three times only at 0.02 and $0.05 \mu \mathrm{g}$ $\mathrm{mL}^{-1}$. And we tried different $M$. ulcerans strains and with tenfold concentrated broth culture. The light from AlMu broth reached $>10$ million $\mathrm{RLU} \mathrm{mL}-1$, which indicating more than $10^{8} \mathrm{CFU} \mathrm{mL}-1$. The series of tenfold diluted culture was diluted and plated on drug-free plates for detecting the bacterium density.

Whole-genome sequencing. The genome DNAs of the parent $M$. marinum and the confirmed TB47-resistant mutants were whole-genome sequenced by Beijing Genomics Institute, China. The resulting reads were aligned to the M. marinum genome sequence and compared with that of the parent strain.

\section{Over-expression of $\mathbf{q} c r B$ genes in $\boldsymbol{M}$. marinum and $\boldsymbol{M}$.ulcerans. Three types} ( $q c r B^{\text {wild type }}, q c r B^{\text {Thr323Pro }}$, and $q c r B^{\text {Thr323Ala }}$ ) of $q c r B$ genes were amplified from genome DNAs of wild-type and spontaneous-resistant $M$. marinum mutants by PCR using primers qcrBmrEf- qcrBmrEr (Table S5) and inserted into the p60luxn plasmid under the control of the hsp 60 promoter. The three plasmids were transformed into the wild-type autoluminescent $M$. marinum or M. ulcerans. The $\mathrm{MICs}_{\mathrm{lux}}$ of the recombinant strains to TB47 were determined as described above.

Pharmacokinetics. BALB/c mice were given $2 \mathrm{mg} \mathrm{kg}^{-1} \mathrm{~TB} 47$ intravenously or 10 $\mathrm{mg} \mathrm{kg}^{-1}$ orally. Blood samples were taken from the eyeballs of five mice per time point at 2, $30 \mathrm{~min}, 1,2,6,12,24$, and $48 \mathrm{~h}$ post-dose for intravenous group or at 15 , $30 \mathrm{~min}, 1,2,6,12,24$, and $48 \mathrm{~h}$ for oral group. Footpads and lung tissues were collected at $6,12,24$, and $48 \mathrm{~h}$ post-dose for oral group. After extraction and centrifuge at $4{ }^{\circ} \mathrm{C}$, TB47 concentrations were determined by Liquid Chromatograph-Mass Spectrometer (LC-MS).

Construction of the $\boldsymbol{M}$. smegmatis cydAB knockout mutant. The recombineering method was used 22,23 . The DNA fragments of upstream of $c y d A$ and downstream of $c y d B$ of M. smegmatis were amplified using primers cydAf-cydAr and cydBfcydBr (Supplementary Table 5) and cloned into a plasmid by three fragment ligation and verified by sequencing. Then the fragment dif-Hyg-dif was inserted in between the two fragments and the resulting ArmLcydA-dif-Hyg-dif-ArmRcydB was excised from the plasmid and transformed into induced M. smegmatis-TS53 (M. smegmatis containing pJV53Ts) competent cells ${ }^{22}$. The $c y d A B$ genes were replaced by the $H y g$ gene through allelic replacement. To remove the Hyg gene, the mutants were cultured in $7 \mathrm{H} 9$ broth without hygromycin for 3 days. To remove vector pJV53Ts, the mutants were cultured in $7 \mathrm{H} 9$ broth at $42^{\circ} \mathrm{C}$ for 3 days, serially tenfold diluted and plated onto $7 \mathrm{H} 11$ plates containing $10 \%$ sucrose, and incubated at $42{ }^{\circ} \mathrm{C}$ for $72 \mathrm{~h}$. The loss of the vector pJV53Ts in the mutants was subsequently confirmed by plating 100 colonies in $7 \mathrm{H} 11$ plates containing $10 \%$ sucrose and kanamycin or in $7 \mathrm{H} 11$ plates containing $10 \%$ sucrose at $42{ }^{\circ} \mathrm{C}$. The M. smegmatis $\triangle c y d A B$ mutant (Msm $\triangle c y d A B)$ was verified by PCR similar to the published design ${ }^{22}$ using primers cyda-d (Supplementary Table 5) and sequencing.

CydAB complementation and growth curves comparison. Complementation plasmids were created by amplifying the $c y d A B$ genes from genome DNAs of $M$. tuberculosis $\mathrm{H} 37 \mathrm{Rv}$, M. ulcerans, M. marinum, and M. smegmatis by PCR using primers indicated in Supplementary Table 5 and inserting them into the p60luxn plasmid under the control of the $h s p 60$ promoter, resulting in the series of plasmids p60luxn-cydAB. A series of recombinant $M$. smegmatis strains were constructed as: $\operatorname{Msm} \triangle c y d A B:: c y d A B$ from M. tuberculosis, Msm $\triangle c y d A B:: c y d A B$ from M. ulcerans, 
$\mathrm{Msm} \triangle c y d A B:: c y d A B$ from M. marinum, Msm $\triangle c y d A B::$ cydAB from M. smegmatis and $\mathrm{Msm} \triangle c y d A B::$ empty vector p60luxn. Log-phase $\left(\mathrm{OD}_{600}=0.5-0.7\right)$ recombinant $M$. smegmatis cultures grown from single colonies were ten thousand-fold diluted and $\mathrm{OD}_{600}$ values of the wild-type and recombinant $M$. smegmatis strains were monitored simultaneously at about $4 \mathrm{~h}$ intervals till the $\mathrm{OD}_{600}$ reached plateau.

Drug susceptibility comparison of $\boldsymbol{M}$. smegmatis strains. The MICs for wildtype and recombinant $M$. smegmatis strains were determined by using the microplate Alamar blue assay ${ }^{8}$. The log-phase cultures $\left(\mathrm{OD}_{600}=0.6-0.8\right)$ were diluted to $\mathrm{OD}_{600}=0.001\left(\sim 10^{5} \mathrm{CFU} \mathrm{mL}^{-1}\right)$. Antibiotics were dissolved in $0.2 \mathrm{~mL}$ of diluted culture in the 96-well microplates. Then $20 \mu \mathrm{L}$ of $10 \times$ alamar blue solution (Alamar Biosciences/Accumed, Westlake, Ohio) was added into each well starting from $36 \mathrm{~h}$ after incubation of the plates at $37^{\circ} \mathrm{C}$. Fluorescence units (FU) were recorded $4 \mathrm{~h}$ for $M$. smegmatis post-alamar blue addition by using the Envision Multilabel Plate Reader (Perkin-Elmer, Massachusetts, MA, USA) with excitation at $530 \mathrm{~nm}$ and emission at $590 \mathrm{~nm}$. The MIC was defined as the lowest concentration that can inhibit $>90 \%$ growth. Different M. smegmatis strains were cultured until $\mathrm{OD}_{600}$ reached $\sim 1.0$, then $10 \mu \mathrm{L}$ of each strain were plated on the same plates containing different concentrations of TB47 and incubated at $37^{\circ} \mathrm{C}$ for $36 \mathrm{~h}$.

Statistical analysis. Experiments were done from at least three biological repeats when possible. RLU and CFU counts were $\log _{10}$ transformed before analysis and were presented as mean \pm SD. Group means were compared by unpaired Student's $t$-tests. The significance level was set at $P<0.05$. Time-to-swelling curves were calculated using the log-rank test. Correlation analysis was performed using Pearson's correlation test. All statistical tests were performed with Graphpad Prism 7 software.

Reporting summary. Further information on experimental design is available in the Nature Research Reporting Summary linked to this article.

\section{Data availability}

Sequence data that support the findings of this study has been deposited in GenBank with accession codes QcrBs (M. tuberculosis: AJF03548.1; M. smegmatis: AFP40620.1; M. marinum: ACC41667.1; M. ulcerans: ABL05699.1) CydAs (M. tuberculosis: CCP44387.1; M. smegmatis: AAF06811.1; M. marinum: ACC40876.1; M. ulcerans: BAV41839.1) CydBs (M. tuberculosis: CCP44386.1; M. smegmatis: AAF06812.2; M. marinum: ACC40875.1; M. ulcerans: BAV41840.1), respectively. The authors declare that all other relevant data supporting the findings of this study are available within the article and its supplementary information files and from the corresponding author upon reasonable request.

Received: 11 November 2018 Accepted: 19 December 2018 Published online: 31 January 2019

\section{References}

1. Van der Werf, T. S., Van der Graaf, W. T., Tappero, J. W. \& Asiedu, K. Mycobacterium ulcerans infection. Lancet 354, 1013-1018 (1999).

2. George, K. M. et al. Mycolactone: a polyketide toxin from Mycobacterium ulcerans required for virulence. Science 283, 854-857 (1999).

3. Etuaful, S. et al. Efficacy of the combination rifampin-streptomycin in preventing growth of Mycobacterium ulcerans in early lesions of Buruli ulcer in humans. Antimicrob. Agents Chemother. 49, 3182-3186 (2005).

4. Converse, P. J. et al. Efficacy of rifampin plus clofazimine in a murine model of Mycobacterium ulcerans disease. PLoS Negl. Trop. Dis. 9, e0003823 (2015).

5. Chauffour, A., Robert, J., Veziris, N., Aubry, A. \& Jarlier, V. Sterilizing activity of fully oral intermittent regimens against Mycobacterium ulcerans infection in mice. PLoS Negl. Trop. Dis. 10, e0005066 (2016).

6. Zhang, T., Li, S. -Y., Converse, P. J., Grosset, J. H. \& Nuermberger, E. L. Rapid, serial, non-invasive assessment of drug efficacy in mice with autoluminescent Mycobacterium ulcerans infection. PLoS Negl. Trop. Dis. 7, e2598 (2013).

7. Zhang, T., Bishai, W. R., Grosset, J. H. \& Nuermberger, E. L. Rapid assessment of antibacterial activity against Mycobacterium ulcerans by using recombinant luminescent strains. Antimicrob. Agents Chemother. 54, 2806-2813 (2010).

8. Tang, J. et al. Design, synthesis, and biological evaluation of pyrazolo [1, 5-a] pyridine-3-carboxamides as novel antitubercular agents. ACS Med. Chem. Lett. 6, 814-818 (2015).

9. Zhang, T. \& Yang, L. New use of pyridine derivatives. International patent application PCT (CN2018/077992) (2018).
10. Dega, H. et al. Bactericidal activity of rifampin-amikacin against Mycobacterium ulcerans in mice. Antimicrob. Agents Chemother. 46, 3193-3196 (2002).

11. Kalia, N. P. et al. Exploiting the synthetic lethality between terminal respiratory oxidases to kill Mycobacterium tuberculosis and clear host infection. Proc. Natl Acad. Sci. USA 114, 7426-7431 (2017).

12. Pethe, K. et al. Discovery of Q203, a potent clinical candidate for the treatment of tuberculosis. Nat. Med. 19, 1157 (2013).

13. Marsollier et al. Isolation of three Mycobacterium ulcerans strains resistant to rifampin after experimental chemotherapy of mice. Antimicrob. Agents Chemother. 47, 1228-1232 (2003).

14. Ji, B., Chauffour, A., Andries, K. \& Jarlier, V. Bactericidal activities of R207910 and other newer antimicrobial agents against Mycobacterium leprae in mice. Antimicrob. Agents Chemother. 50, 1558-1560 (2006).

15. Honrado, E. R., Tallo, V., Balis, A. C., Chan, G. P. \& Cho, S. N. Noncompliance with the world health organization-multidrug therapy among leprosy patients in Cebu, Philippines: its causes and implications on the leprosy control program. Dermatol. Clin. 26, 221-229 (2008).

16. Kuipers, $\mathrm{P}$. What stops people completing multi-drug therapy? Ranked perspectives of people with leprosy, their head of family and neighboursacross four Indian states. Lepr. Rev. 86, 6-20 (2015)

17. Safarian, S. et al. Structure of a bd oxidase indicates similar mechanisms for membrane-integrated oxygen reductases. Science 352, 583-586 (2016).

18. Zhang, T., Li, S. Y. \& Nuermberger, E. L. Autoluminescent Mycobacterium tuberculosis for rapid, real-time, non-invasive assessment of drug and vaccine efficacy. PLoS ONE 7, e29774 (2012).

19. Andréjak, C. et al. Improving existing tools for Mycobacterium xenopi treatment: assessment of drug combinations and characterization of mouse models of infection and chemotherapy. J. Antimicrob. Chemother. 68, 659-665 (2013).

20. Tyagi, S. et al. Bactericidal activity of the nitroimidazopyran PA- 824 in a murine model of tuberculosis. Antimicrob. Agents Chemother. 49, 2289-2293 (2005).

21. Kling, A. et al. Targeting DnaN for tuberculosis therapy using novel griselimycins. Science 348, 1106-1112.14 (2015).

22. Liu, T. et al. Role of folP1 and folP2 Genes in the Action of Sulfamethoxazole and Trimethoprim against Mycobacteria. J. Microbiol. Biotechnol. 25, 1559-1567 (2015).

23. Van Kessel, J. C. \& Hatfull, G. F. Recombineering in Mycobacterium tuberculosis. Nat. Meth. 4, 147 (2007).

\section{Acknowledgements}

This work was supported by the National Mega-project of China for Innovative Drugs (2018ZX09721001-003-003) and for Main Infectious Diseases (2017ZX10302301003-002), by the Chinese Academy of Sciences Grants (154144KYSB20150045, YJKYYQ20170036) and by Guangzhou Municipal Industry and Research Collaborative Innovation Program (201508020248, 201604020019). T.Z. received support "Science and Technology Innovation Leader of Guangdong Province (2016TX03R095)". C.C., G.M., G.C., and M.M.I received the CAS-TWAS Scholarship. H.M.A.H. received the UCAS Scholarship. The founders had no role in study design, data collection and analysis, decision to publish, or preparation of the manuscript. We thank Professor Eric L. Nuermberger at Johns Hopkins University for providing us M. ulcerans strains as kind gifts for our study and Professor Gyanu Lamichhane at Johns Hopkins University for critical reading the manuscript and giving us good suggestions. We thank Dr. Xiantao Zhang, the board chairman of Guangzhou Eggbio Co. Ltd for providing us with TB47 for the experiments. We also thank Professor Chao Zhuo at Guangzhou Medical University for providing us some clinical important strains as kind gifts for our study.

\section{Author contributions}

T.Z. supervised the project, T.Z., Y.L., Y.G, J.L. and Y.T. designed the whole project and most of the experiments, Y.L., Z. Liu, Z. Lu, Y.G., G.M., C.C, S.W., M.M.I. and H.J. performed the susceptibility testing and time-killing curves experiments and Y.L., Y.G., C.C., S.W., Z. Liu, Z. Lu, C.W. and H.J. performed the mechanism of action studies and target validation, Y.L., G.C., Z. Liu, Y.G. and S.W. performed in vivo efficacy experiments, Y.L., Y,G., S.W., C.W., G.M. and H.J. performed drug-resistant mycobacteria screening, verification and the whole-genome sequencing experiments, Z. Liu, Y.T., X.C., X.L., S.W., Z. Lu., J.L., H.M.A.H. and M.M.I. tested TB47 against a panel of microorganisms, T.Z., S.T., and J.L. provided the stains and according reagents, T.Z.,Y.L.,Y.G. and Z. Liu performed bioinformatics analysis, T.Z., Y.L. and Y.G. wrote the manuscript with contributions from other authors.

\section{Additional information}

Supplementary Information accompanies this paper at https://doi.org/10.1038/s41467019-08464-y. 
Competing interests: All authors declare competing interests: TB47 was synthesized in a batch and supplied by Guangzhou Eggbio Co. Ltd, which has been developing TB47 as a therapeutic agent against tuberculosis and other potential diseases and had no role in study design, data collection and analysis, decision to publish the manuscript. Guangzhou Institutes of Biomedicine and Health (GIBH), Chinese Academy of Sciences (CAS) has filed a Chinese application (filing \# 201810106538.9) and a PCT application (filing \# CN2018/077992), entitled 'New use of a set of pyridine compounds', listing T.Z. and Y.L. as inventors.

Reprints and permission information is available online at http://npg.nature.com/ reprintsandpermissions/

Journal Peer Review Information: Nature Communications thanks the anonymous reviewers for their contributions to the peer review of this work

Publisher's note: Springer Nature remains neutral with regard to jurisdictional claims in published maps and institutional affiliations. (c) (i) Open Access This article is licensed under a Creative Commons Attribution 4.0 International License, which permits use, sharing, adaptation, distribution and reproduction in any medium or format, as long as you give appropriate credit to the original author(s) and the source, provide a link to the Creative Commons license, and indicate if changes were made. The images or other third party material in this article are included in the article's Creative Commons license, unless indicated otherwise in a credit line to the material. If material is not included in the article's Creative Commons license and your intended use is not permitted by statutory regulation or exceeds the permitted use, you will need to obtain permission directly from the copyright holder. To view a copy of this license, visit http://creativecommons.org/ licenses/by/4.0/.

(C) The Author(s) 2019 